\title{
Las Mujeres en los Equipos de Gobierno en la Universidad Española. Un Estudio Discreto
}

\section{Women in Government Teams at the Spanish University. An Unobtrusive Study}

\author{
Itziar Rekalde * \\ Esther Cruz Iglesias
}

Universidad del País Vasco/Euskal Herriko Unibertsitatea

\begin{abstract}
Procurar la igualdad de género en el sistema universitario no es sólo una cuestión de justicia social o de ejercicio democrático, sino que es un indicador de excelencia en la comunidad científica. La reciente irrupción de las mujeres en el escenario de la gobernanza universitaria está generando múltiples investigaciones. Si bien hasta ahora las cifras y los porcentajes nos han servido para situar en términos de cantidad a las mujeres en el escenario académico, quizá nos sirvan cada vez menos para explicar un fenómeno que debe de indagarse no sólo desde lo cuantitativo sino también desde la óptica de los matices y sutilezas. Este trabajo profundiza, en términos cuantitativos, en el ámbito de la gestión académica universitaria para desvelar las áreas de desempeño que las mujeres, en cargos académicos por designación (equipos de gobierno), desarrollan en la Universidad pública española. En este estudio exploratorio se utilizan técnicas discretas de naturaleza no reactiva y de dominio público. Se pone de relieve que el 40,91\% de los cargos por designación son ocupados por mujeres y que la distribución, en función de las áreas de desempeño, es desigual. Se expone la necesidad de ofrecer información directa que permita identificar los parámetros sutiles que aún marcan la diferencia entre mujeres y hombres en la distribución de las áreas de desempeño de la gobernanza universitaria.
\end{abstract}

Descriptores: Mujer, Igualdad de oportunidades, Universidad, Administración pública, Gestión de la educación.

Searching for gender equality in the university system is not only a question of social justice or democratic exercise, but an indicator of excellence in the scientific community. The recent emergence of women in the university governance arena is generating multiple investigations. Although up to now the figures and percentages have served to place women in terms of quantity in the academic arena, they may serve us less and less to explain a phenomenon that must be investigated not only from the quantitative but also from the optics of nuances and subtleties. This work delves in quantitative terms in the field of university academic management to reveal the areas of performance that women in academic positions by designation (government teams) develop in the Spanish public university. For this, an exploratory study is carried out using discrete techniques of a non-reactive nature and in the public domain. It is emphasized that $40.91 \%$ of designation fees are occupied by women and that the distribution, according to performance areas, is unequal. It is necessary to provide direct information that allows identifying the subtle parameters that still mark the difference between women and men in the distribution of university governance.

Keywords: Women, Equal opportunity, University, Public administration, Educational management.

*Contacto: itziar.rekalde@ehu.eus

ISSN: 2254-3139

www.rinace.net/riejs/

revistas.uam.es/riejs
Recibido: $\quad 16$ de abril 2017

$1^{\text {a }}$ Evaluación: 13 de mayo 2017

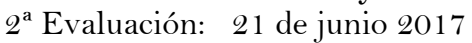

Aceptado: $\quad 3$ de julio 2017 


\section{Revisión de la literatura}

Desde que Concepción Arenal se disfrazó de hombre para poder estudiar en la universidad, la sociedad ha avanzado mucho. Quizá no todo lo que quisiéramos, pero las cifras evidencian ese avance. Si en el curso de 1919/20 sólo había 439 mujeres estudiando en la Universidad española, lo que representaba el $2 \%$ de la población universitaria (Laorden y Giménez, 1978), en el curso de 2014/15 el 53,89\%, en cambio, son mujeres (MECD, 2016).

En ese sentido somos conscientes de que las diferencias de género en las instituciones universitarias están generando un alto incremento de estudios sobre éstas tanto desde su incorporación como alumnas universitarias en los diferentes grados y áreas de conocimiento, como desde la incorporación como docentes e investigadoras $y$, especialmente en la actualidad, por su despliegue en órganos de gestión universitaria. En realidad, procurar la igualdad de género en el sistema universitario no es sólo una cuestión de justicia social o de ejercicio democrático, sino que, tal y como apunta el informe ETAN (2000), es un indicador de excelencia en la comunidad científica.

Si bien hasta ahora las cifras y los porcentajes nos han servido para situar en términos de cantidad a las mujeres en el escenario académico, quizá nos sirvan cada vez menos para explicar un fenómeno que debe de indagarse no sólo desde lo cuantitativo sino también desde la óptica de los matices y sutilezas. Desde una mirada más detallada de la situación de las mujeres en el sistema universitario debemos valorar si el cambio experimentado por las mujeres y los hombres desde principios del siglo XX hasta ahora ha eliminado sustancialmente las desigualdades o sólo las ha transformado. Por ello, moviéndonos en ese terreno de las sutilezas, quizá quede aún por conseguir el tramo más difícil de la igualdad: el de la identificación de mecanismos de discriminación sutiles y poco explícitos.

\subsection{Breve aproximación a la estructura universitaria y al marco legal de igualdad entre hombres y mujeres}

La Ley de Reforma Universitaria (LRU) de 1983 fue la primera ley que configuró la estructura y gobierno de las Universidades españolas adaptándola a los preceptos de la Constitución de 1978. El reconocimiento de la autonomía fue una de sus características fundamentales y alineaba el modelo español con la mayoría de los países desarrollados. En casi todos ellos las Universidades públicas funcionaban respondiendo al modelo colegial, en el que una parte sustancial del gobierno de las Universidades recaía en el personal académico. La LRU concretó los principales órganos de gobierno y dirección de las Universidades (colectivos y unipersonales) (Pérez y Peiró, 1999). Si bien esta Ley fue derogada por la Ley Orgánica de Universidades 6/2001, y ésta a su vez por la Ley orgánica 4/2007, las estructuras principales se siguen manteniendo, de ahí que hablemos del Claustro, Consejo Social y Junta de Gobierno como órganos colectivos y elegidos por la Comunidad universitaria. En cambio, como órganos unipersonales de gobierno para toda la Universidad, se habla de la persona Rectora, de los/las Vicerrectores/as, y del Secretario/a General (artículo 22) y el/la Gerente (artículo 23). Estos cargos son designados directamente por el/la Rector/a quien nombra al Equipo rectoral.

Según Castro e Ion (2011), estos académicos no suelen tener formación en gestión y son apoyados por técnicos administrativos profesionales. Se trata, por tanto, de un modelo de gobernanza no profesional, con algunos rasgos tanto de modelos colegiados como 
democráticos en los que la comunidad académica asume la mayor responsabilidad sobre la toma de decisiones (Castro e Ion, 2011).

En este contexto, desde la entrada en vigor del Tratado de Ámsterdam en la Unión Europea en mayo de 1999, la igualdad de mujeres y hombres se ha convertido en un principio fundamental de la actuación de los poderes públicos. En este tratado aparece expresamente enunciado en su artículo 2 que se persigue la eliminación de las desigualdades, incorporando la perspectiva de género a todas las actuaciones de la Unión. Concretamente el Preámbulo de la Ley Orgánica 4/2007 de 12 de abril, plantea que el reto de la sociedad actual de llegar a una sociedad tolerante e igualitaria, en la que se respeten los derechos y libertades fundamentales y de igualdad entre hombres y mujeres, no se consigue si no se llega a la universidad. Además, su disposición adicional duodécima añade que las universidades contarán entre sus estructuras de organización con unidades de igualdad para desarrollar las funciones relacionadas con el principio de igualdad entre mujeres y hombres. De ahí que los planes de igualdad de las diferentes universidades sean un instrumento para establecer mecanismos y desarrollar acciones que pongan en marcha los diferentes mandatos que recoge el marco legal en relación a esta cuestión.

Esta misma Ley 4/2007, en el apartado 4 del artículo 41, expresa que se debe promover que los equipos de investigación desarrollen su carrera profesional fomentando una presencia equilibrada entre mujeres y hombres en todos sus ámbitos. Así como la Ley 14/2011, de 1 de junio, que en su artículo 2.k) detalla que se promoverá una presencia equilibrada de mujeres y hombres en el Sistema Español de Ciencia, Tecnología e Innovación. En definitiva, se refleja el sentido del término de paridad, entendida como la participación equilibrada de mujeres y hombres en las posiciones de poder y de toma de decisiones en todas las esferas de la vida. Este principio establece que del conjunto de posiciones a repartir (lista electoral, consejo de administración, órgano de gobierno colegiado...) ninguno de los dos sexos tendría que tener una proporción inferior al 40\% ni superior al 60\% (Recomendación 96/694 del Consejo, de 2 de diciembre de 1996, DO L319, citada por Comisión Europea, 1998).

\subsection{Las académicas en la universidad pública española. Una aproximación en cifras}

El incremento significativo del acceso de las mujeres como académicas a la Educación Superior comenzó a finales de los años setenta, y desde entonces las cifras han ido en aumento (Arranz, 2004; García de León y García, 2001). De hecho, la revisión de los datos del Personal Docente e Investigador (PDI) en los últimos diez años facilitada por el MECD (2016) evidencian dicha tendencia (cuadro 1).

Cuadro 1. PDI universitario

\begin{tabular}{cccccc}
\hline CURSO ACADÉMICO & PDI & \% MUJERES & CURSO ACADÉMICO & PDI & \% MUJERES \\
\hline $2004-2005$ & 98.710 & $35,30 \%$ & $2010-2011$ & 115.218 & $38,10 \%$ \\
$2005-2006$ & 101.660 & $35,40 \%$ & $2011-2012$ & 117.527 & Sin información \\
$2006-2007$ & 102.300 & $36,10 \%$ & $2012-2013$ & 115.332 & $39,30 \%$ \\
$2007-2008$ & 105.034 & $36,40 \%$ & $2013-2014$ & 115.071 & $39,90 \%$ \\
$2008-2009$ & 107.930 & $36,60 \%$ & $2014-2015$ & 115.366 & $40,50 \%$ \\
$2009-2010$ & 110.287 & $37,20 \%$ & & & \\
\hline
\end{tabular}

Fuente: MECD. Estadísticas desde el 2006 hasta el 2016. 
Como se puede apreciar en el cuadro 1 el porcentaje de mujeres docentes e investigadoras en la universidad española ha aumentado en estos últimos diez años del $35,3 \%$ en el curso 2004/05 al 40,5\% en el 2014/15.

No obstante, un análisis más pormenorizado de esos porcentajes de mujeres docentes e investigadoras nos puede ayudar a dibujar una forma más definida de ese perfil. De hecho, Sánchez de Madariaga, de la Rica y Dolado (2011) identificaron que el porcentaje de mujeres por categorías académicas y la ratio del número de titulares por cada catedrática eran dos de los principales indicadores que permitían analizar la realidad de las mujeres. Situándonos en los últimos datos que nos ofrece el Ministerio que son para el curso 2014/15 (MECD, 2016), éstos desvelan que un 59,5\% del PDI de la universidad española son hombres y un 40,5\% mujeres.

Respecto a las universidades públicas este porcentaje se reduce muy levemente en detrimento de las mujeres, siendo en las públicas la presencia de mujeres del 39,9\% frente al $43,9 \%$ de las universidades privadas. En cuanto a la distribución por categorías vemos que el porcentaje de mujeres va disminuyendo conforme subimos en el escalafón profesional (gráfico 1). Del 43,9\% de contratadas, al 40,6\% de Titulares de Escuela Universitaria (TEU), al 39,9 de Titulares de Universidad (TU), un 29,7\% de Catedráticas de Escuela Universitaria (CEU) y, finalmente un 20,8\% de Catedráticas de Universidad (CU) (véase gráfico 1). Por lo tanto, en la actual universidad española las mujeres, en general, están más presentes en las categorías inferiores como son las de contratadas y titulares de escuela. En cambio, los hombres tienen más presencia en categorías superiores como la de titulares de universidad, catedráticos de escuela y catedráticos de universidad, donde se subrayan diferencias en los procesos de promoción, y como ya apuntaban Sánchez de Madariaga, de la Rica y Dolado (2011) más significativas si cabe en las cátedras universitarias.

Esta barrera invisible es lo que se conoce como glass ceiling barriers (techo de cristal), concepto que hace referencia a una barrera que se interpone en la parte alta de la carrera laboral de las mujeres y que tiene las características de ser invisible, acotada y sólida (Morrison, White y Van Velsor, 1987). Invisible porque no es percibida como tal por la mayoría de la sociedad y, porque no existen impedimentos explícitos discriminatorios contra las mujeres, sino que es mayoritariamente producto de determinados comportamientos sociales (Matus-López y Gallego-Morón, 2015).

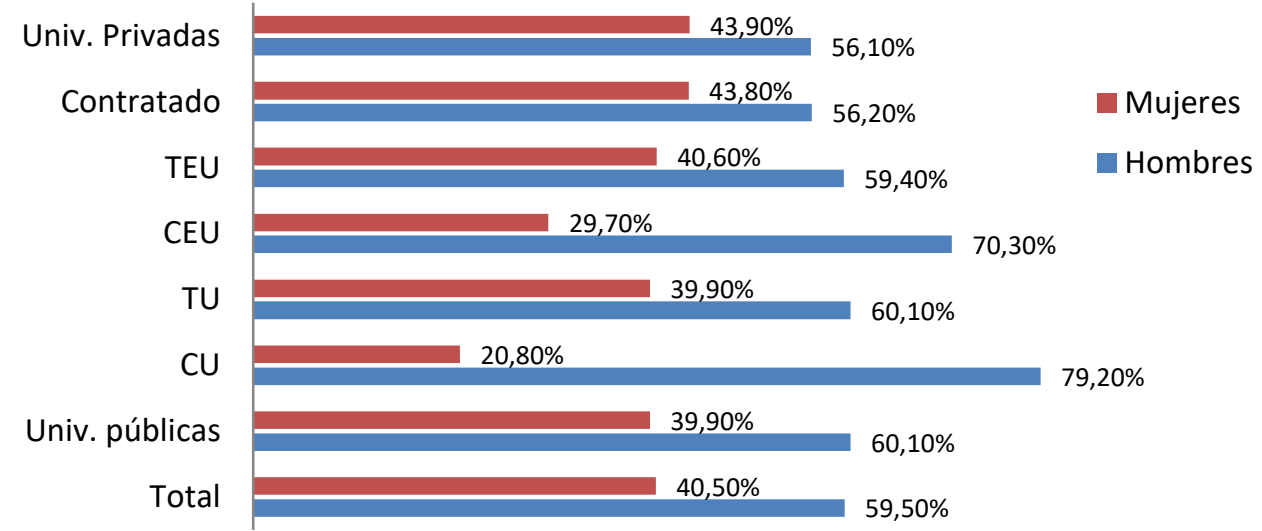

Gráfico 1. Distribución del PDI por sexo y categoría. Curso 2014/15 Fuente: MECD, 2016. Gráfica de diseño propio. 
Cuando la distribución se hace por género y edad (gráfico 2) el porcentaje de mujeres en la universidad va en aumento conforme disminuye la edad. En este caso las mujeres menores de 30 años representan el 60,5\% del PDI de la universidad y en la siguiente franja de edad, de los 30 a los 39 años el porcentaje se equipara con el de los hombres, bajando al 49,8\%. En el siguiente tramo de edad se sigue reduciendo hasta llegar al $43,1 \%$ de mujeres y de ahí el descenso es más acusado; de 50 a 59 años el porcentaje de mujeres es del $37,1 \%$ y de 60 años en adelante del 26,4\%. Como estamos viendo, si bien se están superando las restricciones formales, a la incorporación de las mujeres como PDI en las universidades; se constata que las trayectorias promocionales son diferentes para ambos sexos y los datos ponen en evidencia que se penaliza a las mujeres. Estas compiten curricularmente con los hombres a la hora de acceder a la universidad, pero después, en el caso de que continúen en la carrera universitaria, quedan en el camino de la promoción relegadas en los escalones de menor prestigio (Pastor, 2008; Sánchez de Madariaga, de la Rica y Dolado, 2011). De hecho, hace ya 20 años Sandra Acker (1995) identificó, al menos, tres núcleos problemáticos ante los que se enfrentaban las mujeres académicas: uno, vinculado a las demandas conflictivas de la familia y la carrera profesional, muchas veces difíciles de compatibilizar; dos, la relativa indefensión de las minorías y el dominio que ejercen los hombres sobre el conocimiento y la práctica, y tres, la invisibilidad de las mujeres en los grupos con mayoría masculina donde el liderazgo efectivo se anula.

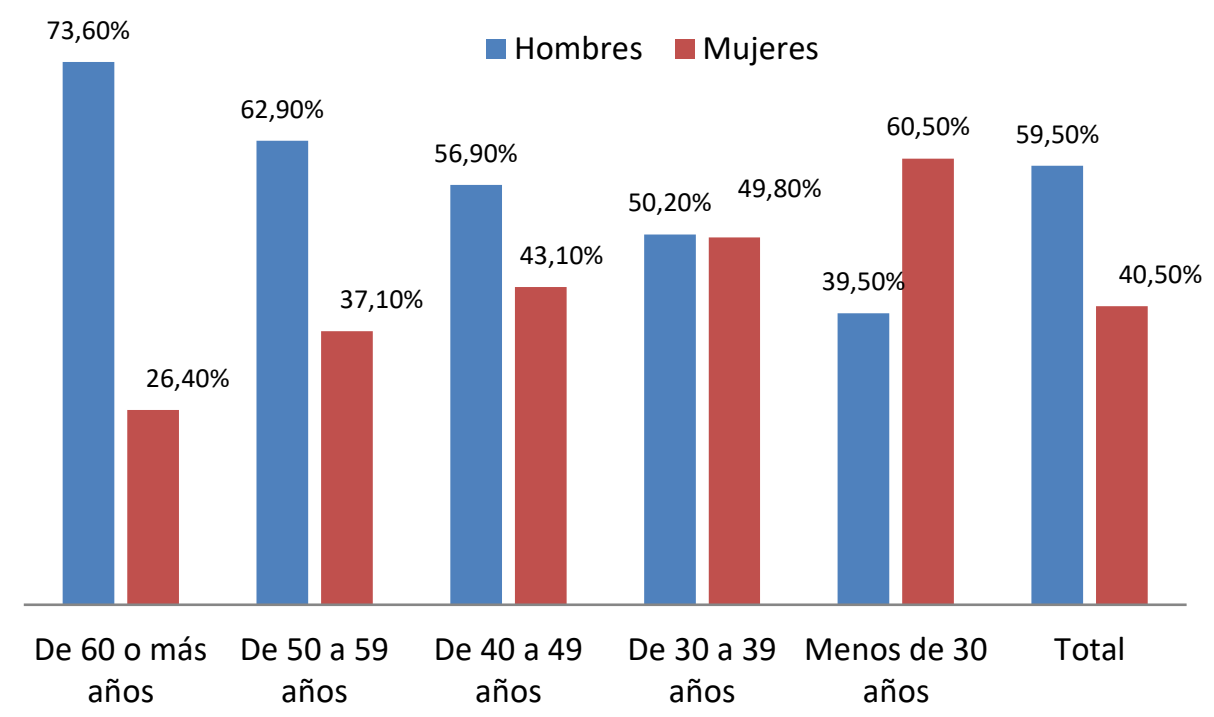

Gráfica 2. Distribución del PDI por sexo y edad. Curso 2014/15 Fuente: MECD, 2016. Gráfica de diseño propio.

Como un indicativo del impacto y desarrollo de la investigación del funcionariado de carrera se asume que son los sexenios óptimos (número de sexenios que dicho personal debiera tener desde la lectura de tesis), y como vemos en el gráfico 3, en el caso de las mujeres catedráticas el 64\% se encuentra en esta situación óptima frente al 61,9\% de los catedráticos hombres. Dato este muy interesante que siguiendo los estudios de Lyness y Heilman (2006) puntualizan que tras la barrera de las dificultades, hay mujeres que se promocionan a costa de mostrar mejores resultados productivos que sus pares varones, $\mathrm{y}$ según Adams y Funk (2012) adoptando comportamientos diferentes al resto de mujeres 
para adaptarse a un medio masculinizado. En cambio, no es esta la tendencia en los cuerpos de Titular de Universidad y Catedrático de Escuela Universitaria, donde el porcentaje de mujeres con sexenios óptimos es inferior; en las Titulares del 38,3\% frente a los $44,7 \%$ de los hombres y en el caso de los CEU las mujeres poseen sexenios óptimos en un $14,5 \%$ frente al $17,6 \%$ de los hombres. Datos que corroboran, nuevamente, una promoción académica más lenta y menos exitosa por parte de las mujeres (Sánchez de Madariaga, de la Rica y Dolado, 2011). Una realidad que se viene interpretando como el dominio de la ciencia y la investigación por parte de los hombres y desde su visión androcéntrica de la realidad y el conocimiento científico (Arranz, 2004; García, Jiménez y Martínez, 2010).

Como ya concluían Sánchez de Madariaga, de la Rica y Dolado (2011) y corroboran los datos del MECD (2016) los hombres promocionan más que las mujeres y la obtención del doctorado no ofrece las mismas posibilidades a mujeres y hombres. En este sentido, diferentes trabajos (Massachusetts Institute of Technology, 1999; Wenneras y Wold, 1997) concluyen que el menor éxito en la universidad por parte de las mujeres no se puede explicar por la falta de productividad científica o académica, dado que, con igualdad de méritos, el puesto de trabajo que obtienen las mujeres es más bajo, y el tiempo medio que tardan en superar un nivel determinado es superior que el de los hombres. En España, en este sentido, la probabilidad de que un profesor titular sea catedrático es de 2,5 veces superior a la de una mujer, aunque tengan características similares (edad, antigüedad como doctores, mismo campo de conocimiento, productividad en artículos y libros...) (gráfico 3). Además, un factor que afecta negativamente a la promoción de las mujeres es el de la maternidad (Acker, 1995). De tal forma que un hombre con hijos tiene cuatro veces más probabilidades que una mujer con hijos y características similares de ser promocionado a catedrático (Sánchez de Madariaga, de la Rica y Dolado, 2011).

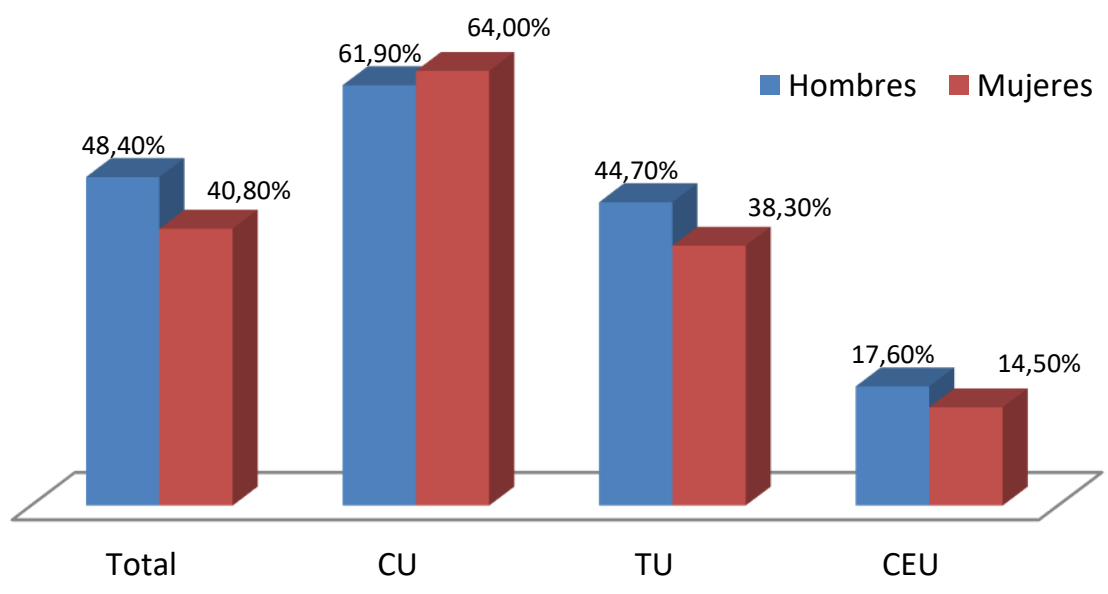

Gráfico 3. Funcionarios de carrera que tienen el número de sexenios que debería tener desde la lectura de tesis (sexenios óptimos) por cuerpo y sexo. Curso 2014/15 Fuente: MECD, 2016. Gráfica de diseño propio.

En 2014 se comprobó que, a pesar de la incorporación de la mujer a la vida laboral universitaria, la presencia de éstas en los máximos órganos de gestión de las universidades públicas y privadas, concretamente en los equipos rectorales seguía sin ser 
paritaria (Almansa, 2014). Se destacó que el vicerrectorado que más comúnmente era ocupado por mujeres era el de Estudiantes y que en las 69 universidades analizadas (públicas y privadas) respecto a las Secretarías Generales casi se había conseguido la paridad entre hombres y mujeres. Así mismo se destacó que el tercer vicerrectorado en el que había más mujeres era el de Ordenación Académica o Docencia, y en el caso del de Investigación, poco menos de un tercio contaba con una mujer al frente.

\subsection{Incorporación de las mujeres a los cargos de gestión. Aproximación a su liderazgo}

Los cambios legislativos y sociales han permitido un incremento de la presencia femenina en los cargos de gestión universitaria, de acuerdo con Breakwell y Tytherleigh (2008), ha avanzado desde un 9,5\% en 1986 a un 23\% en 2006. Asimismo, y de acuerdo con el MEC (2007), hace 10 años la presencia de las mujeres en cargos académicos era escasa dado que había una representación de mujeres inferior al $26 \%$ en los órganos unipersonales en las universidades públicas españolas. Los datos más relevantes correspondían a los referidos a las mujeres que ocupaban los rectorados (sólo un 6,5\% de los casos) o los referidos a la dirección de las facultades o escuelas politécnicas superiores $(16,4 \%)$ mientras que la dirección de departamentos asumida por mujeres era de 19,3\%. El mencionado estudio expresaba que la política de igualdad no reflejaba en los equipos de gobierno de las Universidades públicas españolas, o lo que es lo mismo que existía aún una importante brecha entre la proporción de hombres y de mujeres académicosgestores. Además, esta diferencia se ampliaba al tener en cuenta la manera en que se accede a estas posiciones, porque mientras que los hombres tienden a asumir cargos electivos, las mujeres ocupan en mayor proporción las posiciones designadas por los hombres electos (MEC, 2007).

No obstante, como aluden Airini y sus colaboradores (2011), la llegada de las mujeres a posiciones de poder en las organizaciones es importante en tanto que representa una oportunidad de re-pensar las organizaciones como lugares de trabajo desde una perspectiva de género. De hecho, distintas teorías sobre las organizaciones han trabajado en pro de hacer más visible al género en el campo de la gestión. Según Lavié (2009), primero encontramos perspectivas con un fuerte foco en la demanda de equidad entre hombres y mujeres en el acceso a las posiciones de liderazgo, relacionadas con la supresión de las barreras institucionales que dificultan el acceso, y un segundo grupo subraya la gestión como un campo ajeno a la mayoría de las mujeres, en el que éstas tienen una particular contribución que hacer (citado en Sánchez-Moreno, López-Yáñez y Altopiedi, 2014).

Desde este último grupo se expone que los hombres tienden a poner en juego un estilo de liderazgo basado en las cualidades instrumentales (asertividad, control, ambición, competitividad, independencia y confianza), mientras que las mujeres adoptan uno basado en las cualidades emocionales (escucha y apoyo a las personas) dirigido al bienestar de los demás y hacia la resolución de los conflictos interpersonales (Eagly y Johannesen-Schmidt, 2001; Eagly, Wood y Diekman, 2000). Hecho este que se corrobora en el estudio de Sánchez-Moreno, López-Yáñez y Altopiedi (2014), donde se concluye que las mujeres gestoras en la universidad no despliegan un estilo de liderazgo radicalmente distinto del de los hombres, pero sí que muestran sutiles diferencias en el alto grado en que éstas priorizan la atención a las personas, el bienestar del grupo y los aspectos informales de la gestión, mientras que los gestores varones tienden a priorizar 
la toma de decisiones y el cumplimiento de las tareas. Por lo tanto, la literatura reconoce la habilidad en las relaciones sociales como un rasgo que define un perfil predominante de liderazgo femenino, en contraste, con el de los varones gestores que emplean en mayor medida rasgos como la habilidad para conseguir recursos y tomar decisiones.

Teniendo en cuenta todo lo dicho hasta ahora el presente estudio realiza un acercamiento actualizado desde la perspectiva de género (dado que las cifras están en constante cambio), respecto a la presencia de mujeres en los equipos de gobierno (cargos unipersonales) en el escenario universitario. Para ello los objetivos que se plantean son dos; uno, cuantificar la representación de mujeres en cargos unipersonales (Vicerrectorados, Secretaría general y Gerencia) en la universidad pública española, y dos; analizar la distribución de mujeres en función de las áreas de desempeño. Digamos que, tras la revisión de la literatura, se parte de la hipótesis de que la presencia de mujeres en el escenario de la gobernanza universitaria ha aumentado respecto a estudios anteriores y, que la distribución de éstas en las diferentes áreas de la gestión universitaria es proporcional.

\section{Método}

Ha consistido en un estudio exploratorio dado que el objeto de estudio se ha centrado, básicamente, en examinar la representación que las mujeres tienen en los equipos de gobierno de las universidades públicas españolas, tema o situación de investigación que requiere constante revisión e interpretación. Como apuntan Hernández, Fernández y Baptista (2003) los estudios exploratorios nos sirven para aumentar el grado de familiaridad con fenómenos relativamente desconocidos del cual se tienen muchas dudas, obtener información sobre la posibilidad de llevar a cabo una investigación más completa, establecer prioridades para investigaciones posteriores, etc. En este mismo sentido Ferreyra y De Longhi (2014) sostienen que en el caso de que el problema planteado no haya sido abordado anteriormente o esté poco estudiado, es aconsejable optar por un estudio de tipo exploratorio que permita lograr un contacto con la realidad que luego se estudiará de forma más profunda. Por tanto, es un estudio exploratorio en su dimensión cronológica (debido a que los nombramientos están o pueden estar en constante cambio), interpretativa (escasos estudios previos en relación al objeto concreto de estudio), y de profundización (con la intención de seguir indagando con instrumentos de investigación intrusivos).

Teniendo en cuenta este carácter exploratorio y siguiendo a Sabariego-Puig, MassotLafon y Dorio-Alcaraz (2009) el método idóneo a utilizar para alcanzar la finalidad planteada es la teoría fundamentada que, según las autoras, es de naturaleza exploratoria y su propósito el descubrir teorías, conceptos, hipótesis y proposiciones partiendo directamente de los datos de manera inductiva. Su validez radica al ser aplicado en ámbitos nuevos de estudio donde hacen falta conceptos fundamentados para describir y explicar lo que sucede.

Al ser este estudio una primera aproximación se han utilizado técnicas de acceso a los datos denominadas discretas, que tratan de hallar maneras indirectas de obtener los datos necesarios para realizar una primera aproximación al objeto de estudio. Las medidas discretas pueden ser fuentes de datos inusuales (grafitis, basura...) o el propio espacio virtual (webs y huellas digitales). Tengamos en cuenta que son instrumentos de 
naturaleza no reactiva porque no involucran a los sujetos de la investigación (Lee, 2000; Webb et al., 2000) y que, como es nuestro caso, son datos de dominio público y, por lo tanto, se preservan cuestiones relacionadas con la ética en la investigación. En este sentido, el estudio utiliza como medida discreta de información las páginas web corporativas de todas las universidades españolas (revisión llevada a cabo durante el mes de marzo de 2017).

En cuanto al procedimiento analítico se ha desarrollado de forma concomitante al trabajo de campo (Goetz y Lecompte, 1988), en el que el análisis de la información obtenida ha representado un proceso, básicamente inductivo, dinámico y sistemático que ha requerido identificar, seleccionar, categorizar, comparar e interpretar el mensaje de la información obtenida en la red (Coffey y Atkinson, 2003).

$\mathrm{Al}$ objeto de poder analizar esta información se han creado dimensiones a través de las cuales ir categorizando la información relativa a las áreas de desempeño de los diferentes vicerrectorados. En este sentido, debemos puntualizar que si bien hay cargos que la Ley orgánica 4/2007 establece como obligatorios (Rector/a, Secretario/a General y Gerente/a), respecto a los vicerrectorados cada universidad dispone y nombra sus propias unidades. Dada la complejidad del contexto de estudio, el trabajo se ha centrado en las unidades designadas por las propias universidades como vicerrectorados y no en direcciones o servicios que, en ocasiones, aparecen en las páginas web junto a los vicerrectorados. En definitiva, el análisis ha pretendido identificar elementos y explorar sus conexiones, teniendo en cuenta que las dimensiones eran excluyentes.

\section{Resultados}

El análisis de los datos nos muestra que de las 76 universidades españolas que actualmente existen (datos de marzo de 2017), en 9 de ellas está una Rectora al mando de la institución, lo que significa que el 11,8\% de las universidades españolas están lideradas por mujeres.

Si diferenciamos universidades públicas y privadas, en este caso, los datos nos revelan que de las 50 universidades públicas que hay actualmente en España, en 3 de ellas la Comunidad universitaria ha nombrado como Rectora a una mujer (Universidad de Granada, Universidad Autónoma de Barcelona y Universidad del País Vasco/Euskal Herriko Unibertsitatea), lo que significa que las cifras en términos de porcentajes son de un $6 \%$. Respecto a las universidades privadas, de las 26 que existen en la actualidad, en 6 de ellas hay mujeres nombradas como Rectoras (Universidad Cardenal Herrera, Universidad Católica San Antonio de Murcia, Universidad Católica de Ávila, Universidad a Distancia de Madrid, Universidad Europea Miguel de Cervantes y Universidad Pontificia de Salamanca), lo que en términos porcentuales significa un $23 \%$.

\subsection{Las mujeres en los equipos de gobierno universitarios}

Exponemos a continuación los resultados obtenidos en relación a la presencia de mujeres en los equipos de gobierno de las universidades públicas españolas (cuadro 2). Cargos unipersonales que son designados por la persona que ejerce el cargo máximo de gobernanza en la universidad: el Rector o Rectora.

Cuadro 2. Presencia de mujeres en los Vicerrectorados, Gerencia y Secretaría general en cada universidad pública española 


\begin{tabular}{|c|c|c|c|c|}
\hline UNIVERSIDAD & $\begin{array}{c}\text { NÚM. DE } \\
\text { VICERREC- } \\
\text { TORADOS }\end{array}$ & $\begin{array}{l}\text { VICERREC- } \\
\text { TORAS }\end{array}$ & $\begin{array}{c}\text { LA } \\
\text { GERENTE }\end{array}$ & $\begin{array}{c}\text { LA } \\
\text { SECRETARIA }\end{array}$ \\
\hline U. Autónoma de Barcelona & 10 & 2 & & * \\
\hline U. Autónoma de Madrid & 9 & 4 & & * \\
\hline U. Carlos III Madrid & 7 & 4 & & \\
\hline U. Complutense de Madrid & 12 & 6 & & * \\
\hline U. de A Coruña & 7 & 4 & & \\
\hline U. de Alcalá & 8 & 2 & & \\
\hline U. de Alicante & 10 & 4 & & * \\
\hline U. de Almería & 6 & 2 & * & \\
\hline U. de Barcelona & 10 & 4 & & * \\
\hline U. de Burgos & 8 & 3 & & \\
\hline U. de Cádiz & 8 & 2 & & * \\
\hline U. de Cantabria & 8 & 3 & & * \\
\hline U. de Castilla-La Mancha & 8 & 5 & & \\
\hline U. de Córdoba & 9 & 4 & * & * \\
\hline U. de Extremadura & 9 & 2 & & * \\
\hline U. de Girona & 8 & 2 & * & \\
\hline U. de Granada & 7 & 4 & * & \\
\hline U. de Huelva & 5 & 3 & & \\
\hline U. de Jaén & 8 & 4 & & * \\
\hline U. de La Laguna & 8 & 4 & * & * \\
\hline U. de La Rioja & 5 & 2 & & \\
\hline U. de las Illes Balears & 9 & 4 & * & \\
\hline U. de Las Palmas de Gran Canaria & 10 & 4 & * & * \\
\hline U. de León & 9 & 4 & * & * \\
\hline U. de Lleida & 9 & 2 & & * \\
\hline U. de Málaga & 11 & 5 & * & \\
\hline U. de Murcia & 10 & 4 & & \\
\hline U. de Oviedo & 8 & 2 & & * \\
\hline U. de Salamanca & 8 & 4 & & * \\
\hline U. de Santiago de Compostela & 9 & 4 & & * \\
\hline U. de Sevilla & 9 & 6 & & * \\
\hline U. de Vàlencia & 9 & 3 & & * \\
\hline U. de Valladolid & 12 & 3 & & * \\
\hline U. de Vigo & 7 & 3 & & * \\
\hline U. de Zaragoza & 12 & 5 & & \\
\hline U. del País Vasco/EHU & 11 & 5 & $*$ & \\
\hline U. Internacional de Andalucía & 6 & 2 & & * \\
\hline U. Internacional Menéndez Pelayo & 4 & 2 & * & \\
\hline U. Jaume I Castellón & 9 & 4 & & \\
\hline U. Miguel Hernández de Elche & 11 & 4 & * & * \\
\hline $\begin{array}{l}\text { U. Nacional de Educación a } \\
\text { Distancia }\end{array}$ & 7 & 5 & * & * \\
\hline U. Pablo de Olavide & 10 & 4 & & \\
\hline U. Politécnica de Cartagena & 9 & 1 & * & * \\
\hline U. Politécnica de Catalunya & 8 & 3 & * & \\
\hline U. Politécnica de Madrid & 7 & 1 & & * \\
\hline U. Politécnica de Valencia & 8 & 3 & & \\
\hline U. Pompeu Fabra & 8 & 3 & & * \\
\hline U. Pública de Navarra & 6 & 3 & & * \\
\hline U. Rey Juan Carlos & 10 & 3 & * & $*$ \\
\hline U. Rovira i Virgili & 7 & 3 & & \\
\hline
\end{tabular}

Fuente: Elaboración propia a partir de los datos web de marzo de 2017. 
En cuanto a la figura de la persona Gerente de la universidad, este cargo está ocupado por mujeres en 16 de las 50 universidades públicas (32\%) y en el caso de la figura de la persona Secretaria general de la universidad, las cifras son muy superiores; en 29 de las 50 universidades el puesto está ocupado por una mujer (58\%) (cuadro 3). Téngase en cuenta que estos dos cargos junto con el de Rector/a existen como tal en todas las universidades, por lo que el cálculo de porcentajes es pertinente y ajustado a la realidad.

El Vicerrector/a es la persona de categoría inmediatamente inferior a la de rector/a y puede sustituirlo en determinadas ocasiones. Pues bien, el número total de los diferentes Vicerrectorados que cada universidad establece para el desarrollo de la política universitaria son un total de 423, de los cuales 169 están ocupados por mujeres, lo que significa que, aproximadamente, un 40\% (39,95\%) de los vicerrectorados de las distintas universidades públicas españolas están ocupados por mujeres en la actualidad (cuadro 3).

Cuadro 3. Presencia de mujeres en los Equipos de gobierno de la Universidad española

\begin{tabular}{lrcc}
\hline \multicolumn{1}{c}{ CARGO } & TOTAL & MUJERES & PORCENTAJE \\
\hline Rector/a & 50 & 3 & $6 \%$ \\
Secretario/a general & 50 & 29 & $58 \%$ \\
Gerente/a & 50 & 16 & $32 \%$ \\
Vicerrectores/as & 423 & 169 & $39,95 \%$ \\
\hline
\end{tabular}

Fuente: Elaboración propia a partir de los datos web de marzo de 2017.

Esto significa que en su conjunto y por lo que se refiere a los cargos unipersonales por designación (Secretaría general, Gerente y Vicerrectores/as) se llega al 40,91\% de mujeres, y cuando hablamos de Equipos de gobierno, donde quien preside dicho equipo es quien ejerce de Rector/a el porcentaje disminuye al 37,87\% de mujeres.

\subsection{La distribución de las mujeres en función de las áreas de gobernanza}

En cuanto a la distribución de las mujeres vicerrectoras en función de las grandes dimensiones que se han construido para acoger las amplias áreas de gestión universitaria, digamos que, en todas las universidades con posibilidad de variantes nominales, se pueden identificar las siguientes: Alumnado, Profesorado (PDI), Estudios (Ordenación académica), Investigación e Internacionalización. Otras dimensiones con mayor variabilidad en la denominación y en las tareas a desempeñar son: Docencia, Vida universitaria y Sociedad, e Infraestructuras.

Los resultados que se derivan de ordenar los vicerrectorados universitarios liderados por mujeres en estas dimensiones que responden a las principales áreas de gestión universitaria pueden verse representadas en el gráfico 4.

Bajo la categoría de Alumnado se han incluido aquellos vicerrectorados que atienden cuestiones relacionadas con el estudiantado, la empleabilidad y el emprendimiento del alumnado universitario. Si bien adquieren diferentes designaciones (alumnado, estudiantes...) pretenden informar, orientar y asesorar de los derechos y recursos que el estudiantado dispone en las respectivas universidades. Unidades que atienden de la orientación preuniversitaria y durante su permanencia en la Universidad; la atención y respuesta a las necesidades educativas especiales del estudiantado, así como información y orientación para la transición al mundo laboral y actuaciones vinculadas a su inserción laboral. 
Son 38 los Vicerrectorados de Alumnado de las 50 Universidades públicas los que están liderados por mujeres. Por tanto, en el $76 \%$ de los vicerrectorados que atienden cuestiones directamente relacionadas con el estudiantado, tenemos al frente a una mujer.

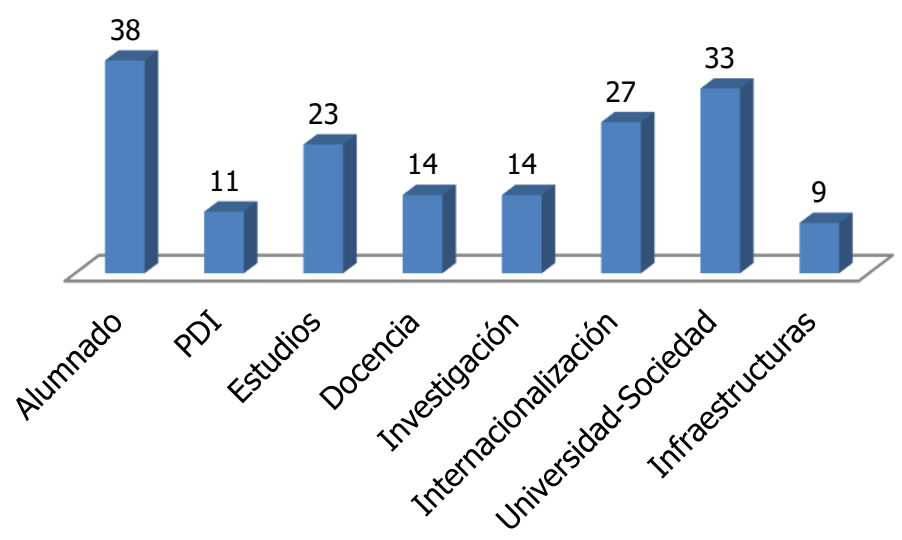

Gráfico 4. Liderazgo femenino en los vicerrectorados de las universidades públicas españolas

Fuente: Elaboración propia a partir de los datos web de marzo de 2017.

En relación a la dimensión denominada PDI (Personal Docente e Investigador) recogemos aquellos vicerrectorados que abordan temas relativos al personal contratado en la universidad para desempeñar labores docentes e investigadoras. En ellos se abordan los temas de selección, formación y promoción; compatibilidades, derechos y obligaciones académicas, administrativas y laborales; Permisos y licencias, retribuciones, nombramiento y contratación, así como la planificación y gestión de la plantilla del personal docente e investigador. En esta área de desempeño son 11 de los 50 vicerrectorados los que están liderados por mujeres; hablamos, por tanto, de un $22 \%$.

La dimensión de Estudios engloba términos como el de Ordenación académica, Oferta académica, Estudios de grado y posgrado... Son los vicerrectorados encargados de la autorización y verificación de los planes de estudio de las titulaciones de Grado y Posgrado; oferta, seguimiento y acreditación de las titulaciones oficiales; convalidaciones de los títulos oficiales y propios de Grado y de Posgrado, expedición de títulos y certificaciones académicas... En esta dimensión las vicerrectoras que desempeñan sus funciones como tal son 23.

En cuanto a la dimensión denominada Docencia se engloban aquellos vicerrectorados que abordan temas vinculados directamente con la docencia, la innovación curricular y docente, y la calidad universitaria. En estos vicerrectorados se desarrollan apoyos a los procesos de enseñanza-aprendizaje, la formación del personal docente e investigador, la implantación y difusión de programas de innovación, gestión de calidad y difusión de resultados y buenas prácticas. Son 14 vicerrectorados los que están liderados por mujeres. Digamos que esta dimensión, aunque pueda tener entidad por sí misma, en ocasiones, las universidades la vinculan a la de Estudios u Ordenación académica. En el caso de fusionar estas dos dimensiones, el segundo ámbito de actuación mayoritariamente femenino sería el de Estudios y Docencia, con 37 mujeres al frente de vicerrectorados de esta naturaleza. 
Respecto a la dimensión denominada Investigación, digamos que junto a Alumnado es la dimensión que más claramente se identifica bajo las denominaciones que las diferentes universidades lo designan y es el vicerrectorado que no falta en ninguna universidad. En este caso bajo esta denominación encontramos también términos como política científica, o transferencia del conocimiento. Las tareas que se desarrollan son el fomento de la actividad investigadora; la participación en convocatorias competitivas promovidas por entidades públicas o privadas, nacionales o internacionales... Bajo esta dimensión encontraríamos a 14 mujeres que lideran este ámbito, o en términos de porcentaje (dado que en todas las universidades existe un vicerrectorado dedicado a estos asuntos); un 28\% de los vicerrectorados que se encargan de la investigación su líder es una mujer.

En cuanto a la dimensión denominada Internacionalización se engloban los vicerrectorados también denominados como Relaciones Internacionales. Sus principales actividades son la colaboración con universidades u entidades extranjeras, la gestión y promoción de acciones de intercambio, la gestión de los programas de movilidad... Son 27 las mujeres que estarían liderando vicerrectorados cuyo objetivo es la internacionalización de su universidad. Siendo un área de desempeño que se encuentra en todas las universidades, digamos que el $54 \%$ de las personas que ocupan estos vicerrectorados son mujeres.

Respecto a la dimensión denominada Vida universitaria y Sociedad se engloban términos como la extensión universitaria, la acción cultura, el deporte, los servicios sociales y comunitarios, la proyección social y comunicación, la inclusión, la igualdad, la responsabilidad social... En este caso 33 vicerrectoras se encargan de estas cuestiones.

Finalmente, la última dimensión es quizá aquella en la que se incluyen términos y áreas de desempeño muy variopintos; asuntos relacionados con las Infraestructuras, la Tecnología, los Campus, la planificación económica... Son 9 las mujeres que se encargan de estas cuestiones en la actualidad.

\section{Discusión y conclusiones}

La igualdad es uno de los derechos fundamentales de todas las personas y constituye la base para el ejercicio de la libertad. A pesar de que existen parámetros políticos e institucionales que rompen con el denominado techo de cristal y, que en cuanto a las cifras se refiere están cumpliendo con su cometido; las diferencias, ya no están en el terreno legal ni en el numérico, sino en el de la igualdad ante las sutilezas y matices con las que se alimenta la sociedad. Es decir, si bien la distancia numérica entre hombres y mujeres es cada vez menor, la persistencia de ámbitos y áreas estereotipados que actúan en paralelo es todavía apreciable. En este trabajo se da cuenta de ello.

Respecto a la cuantificación de la representación de mujeres en cargos unipersonales en los equipos de gobierno en la universidad pública española este trabajo concluye que actualmente las universidades públicas españolas están dirigidas en un 6\% por rectoras. En tres años los datos se han triplicado; de tener una única mujer Rectora a tener tres en la actualidad (marzo de 2017). Si bien la tendencia es al alza la presencia femenina sigue siendo, prácticamente, anecdótica en las universidades públicas (Almansa, 2014). Este hecho se acompasa con las mayores dificultades que las mujeres tienen de promoción y de acceso a cátedra (indispensable para ejercer de Rectora) (Massachusetts Institute of Technology, 1999; Pastor, 2008; Sánchez de Madariaga, de la Rica y Dolado, 2011; 
Wenneras y Wold, 1997). No obstante, los porcentajes nos desvelan que la tendencia de las universidades privadas es de mostrar porcentajes mayores de rectoras frente a las públicas. En este caso, y para marzo de 2017, es del 23\% de rectoras, tendencia que se acompasa también con el número mayor de PDI mujeres en las universidades privadas (MECD, 2016). Aunque esta lectura no es tan alentadora si tenemos en cuenta que la gobernanza de las universidades privadas cuenta con otros cargos (presidentes o cancilleres) que rebajan el poder de quien ejerce de Rector/a.

Sobra decir que para el más alto cargo universitario las medidas paritarias entre hombres y mujeres no han tenido aún unas consecuencias significativas (Comisión Europea, 1998) y que los efectos invisibles del fenómeno denominado Techo de Cristal sigue muy presente en la cúspide de la jerarquía universitaria (Matus-López y GallegoMorón, 2015; Morrison, White y Van Velsor, 1987). No obstante, el panorama conforme bajamos un escalafón en la pirámide de la gobernanza de las universidades públicas varía sustancialmente:

En el caso de la persona que ocupa el cargo de la Secretaria general de la universidad, este estudio muestra que es ocupado en un $58 \%$ por mujeres y que los datos ofrecidos por estudios anteriores nos permiten afirmar que ha ido en aumento (Almansa, 2014). En cambio, la figura de Gerente/a de la universidad está desempeñado por mujeres en un $32 \%$. Lo que significa que desde la perspectiva de género y a la luz de una lectura paritaria (Comisión Europea, 1998; Ley 14/2011), que si bien en el caso de la Secretaria general se cumple con las proporciones justas indicadas por las normativas (entre el $40 \%$ y el $60 \%$ ) no se cumple, en cambio para la figura de quien ocupa la gerencia de la Universidad.

No obstante, esta interpretación aislada no refleja siempre y en todos los casos la realidad de las universidades, dado que se puede obtener un equilibrio entre los datos de estos dos cargos claves en la gobernanza; cuando el cargo de la Secretaría general está ocupado por una mujer el de la Gerencia lo está por un hombre. Esto no quita, sin embargo, que el cargo de la Gerencia no llegue a los porcentajes deseados de paridad, por lo que es un dato que nos puede llevar a la reflexión.

Se concluye, además, que en marzo de 2017 el 39,95\% de los vicerrectorados de las universidades públicas españolas están ocupados por mujeres, y que se llega al 40,91\% de mujeres cuando nos referimos a los cargos unipersonales por designación (Secretaría general, Gerente y Vicerrectores/as). Se aprecia, por tanto, un aumento significativo que se apuntaba por el MEC en 2007. Esto significa que tomando como criterio las disposiciones de la Comisión Europea (1998) se han necesitado casi 20 años para llegar al porcentaje mínimo del $40 \%$ que garantiza la paridad entre mujeres y hombres. La superación de esta barrera, gracias en parte a los Planes de Igualdad de las universidades, refleja mejor la composición de la sociedad en la universidad, y garantiza los intereses de las mujeres en la elaboración de las políticas universitarias. Además, en la línea de Lavie (2009) y Airini y sus colaboradores (2011) representa una oportunidad de re-pensar la organización universitaria desde una perspectiva de género.

En cuanto al análisis de la distribución de mujeres en función de las áreas de desempeño de los vicerrectorados este trabajo concluye que bajo la dimensión denominada Estudios son 23 las mujeres al frente de estos vicerrectorados; es decir, un $46 \%$ de los mismos están liderados por académicas. En cuanto a la dimensión de Internacionalización de la universidad son 27 las mujeres que están en la actualidad liderando vicerrectorados bajo 
esta dimensión; o en otros términos el 54\% de quienes lideran esta área de la gestión universitaria son mujeres. Basándonos en las recomendaciones de la Unión europea y toda la normativa al respecto (Comisión Europea, 1998) estos dos ámbitos de la gobernanza universitaria responden adecuadamente al principio de la presencia equilibrada entre mujeres y hombres.

El vicerrectorado de alumnado es en el que la gobernanza por parte de las mujeres es mayoritaria. En esta dimensión se puede concluir que de las 50 Universidades públicas en 38 es una mujer quien lidera este vicerrectorado (el $76 \%$ ). De hecho, no es un dato nuevo puesto que la preponderancia femenina en los vicerrectorados de alumnado/estudiantes se mantiene desde el 2014 (Almansa, 2014) hasta la actualidad. Debiéramos reflexionar si el hecho de la designación como Vicerrectoras de Alumnado tiene que ver con las cualidades emocionales que ponen en juego dirigidas al bienestar y a las relaciones interpersonales que la literatura revela como características de los estilos de liderazgo de las mujeres (Eagly y Johannesen-Schmidt, 2001; Eagly, Wood y Diekman, 2000; Sánchez-Moreno, López-Yáñez y Altopiedi, 2014). Además, utilizando los parámetros de las normativas europeas en cuestiones de paridad (Comisión Europea, 1998) donde se recomienda que ninguno de los dos sexos tenga una proporción superior al $60 \%$, entre otras cuestiones para evitar percepciones estereotipadas sobre áreas y ámbitos de actuación, vemos que en este caso aunque en positivo por el número de mujeres no se cumple con la finalidad que desde la perspectiva de género se quiere instaurar en todos los órdenes de la vida social, política, económica y, cómo no, también universitaria.

En el área de desempeño encargada de la gestión del Personal docente e investigador de la universidad son 11 de los 50 vicerrectorados los que están liderados por mujeres; hablamos, por tanto, como ya se ha señalado anteriormente de un $22 \%$. Algo similar ocurre con la dimensión denominada investigación donde encontraríamos a 14 mujeres que lideran este ámbito, o en términos de porcentaje; un $28 \%$ de los vicerrectorados que se encargan de la investigación su líder es una mujer. Como se subrayaba con los datos del estudio de Almansa (2014) donde se citaban 19 vicerrectoras de investigación en universidades públicas y privadas, ni era ni es un porcentaje desdeñable dada la importancia del vicerrectorado y dado que ha sido tradicionalmente ocupado por hombres (Almansa, 2014). Sin embargo, los parámetros europeos relativos a la paridad (Comisión Europea, 1998) nos ponen sobre aviso de que los números juegan en negativo para las mujeres. Esto no nos ha de sorprender si tenemos en cuenta que el dominio de los hombres en el ámbito de la ciencia y la investigación ha sido mayoritario (Arranz, 2004; García, Jiménez y Martínez, 2010).

En cuanto a otro ámbito amplio en el que se encuentran las mujeres desarrollando su labor de gobernanza universitaria es en el designado como vida universitaria y sociedad. Es un número alto, 33 vicerrectoras, pero los asuntos que se abordan bajo este ámbito son muy variados y no nos permite obtener porcentajes al respecto. Finalmente son 14 las mujeres que ocupan la gobernanza del ámbito de la Docencia y 9 en el ámbito designado como Infraestructuras, tendencia que se corresponde con estudios anteriores (Almansa, 2014).

Con este trabajo se concluye que la hipótesis inicial se cumple en parte; respecto a la presencia de mujeres en el escenario de la gobernanza universitaria, sí que se constata que ha aumentado desde los estudios más recientes hasta el presente de 2017 que se 
sitúa en un 40,91\% de mujeres en los equipos de gobierno. En cambio, la distribución de las mujeres en las diferentes áreas de la gestión universitaria no es proporcional. Es decir, que la distribución de las mujeres en función de las diferentes áreas de la gestión universitaria no responde a los principios de paridad entre mujeres y hombres sobre todo en los ámbitos relativos a Alumnado, por excederse en los porcentajes recomendados, y en Investigación, por su déficit porcentual. Esto nos lleva indiscutiblemente a asociar los ámbitos a los estereotipos sociales y a las cualidades que se persiguen en cada área. En cambio, en los ámbitos relativos a Estudios e Internacionalización se habrían alcanzado los porcentajes óptimos desde una lectura paritaria y sin vinculaciones estereotipadas desde una perspectiva de género.

Antes de finalizar, debemos apuntar que somos conscientes de las limitaciones que tiene este estudio, entre otras, la falta de triangulación de los datos. Al ser un estudio exploratorio y aproximativo al objeto de estudio se han utilizado medidas discretas para, a partir de los resultados obtenidos, planificar una fase posterior de profundización con instrumentos de tipo más intrusivos (entrevistas, encuestas, grupos de discusión...) que nos ofrezcan una información directa que nos permita identificar los parámetros sutiles que aún marcan la diferencia entre mujeres y hombres en la distribución de las áreas de desempeño de la gobernanza universitaria.

\section{Referencias}

Adams, R. y Funk, P. (2012). Beyond the glass ceiling: Does gender matter? Management Science, 58(2), 219-235. https://doi.org/10.1287/mnsc.1110.1452

Acker, S. (1995). Género y educación. Madrid: Narcea.

Airini, C.S., Conner, L., Mcpherson, K., Midson, B. y Wilson, C. (2011). Learning to be leaders in higher education: What helps or hinders women's advancement as leaders in universities. Educational Management Administration $\& \quad$ Leadership, 39(1), 44-62. https://doi.org/10.1177/1741143210383896

Almansa, A. (2014). La mujer en la gestión universitaria: ¿Paridad o apariencia de paridad? En VVAA., Libro de actas del II congreso internacional de comunicación y género (pp. 654-663). Madrid: Dykinson.

Arranz, F. (2004). Las mujeres y la Universidad española: estructuras de dominación y disposiciones feminizadas en el profesorado universitario. Politica y Sociedad, 41(2), 223242 .

Breakwell, G. y Tytherleigh, M. (2008). UK university leaders at the turn of the 21 st century: Changing patterns in their socio-demographic characteristics. Higher Education, 56, 109127. https://doi.org/10.1007/s10734-007-9092-2

Castro, D. e Ion, G. (2011). Dilemas en el gobierno de las universidades españolas: Autonomía, estructura, participación y desconcentración. Revista de Educación, 355, 161-183.

Coffey, A. y Atkinson, P. (2003). Encontrar sentido a los datos cualitativos. Medellín: Contus.

Comisión Europea (1998). 100 palabras para la igualdad. Glosario de términos relativos a la igualdad entre mujeres y hombres. Luxemburgo: Oficina de Publicaciones Oficiales de las Comunidades Europeas.

Eagly, A. H. y Johannesen-Schmidt, M. C. (2001). The leadership styles of women and men. Journal of Social Issues, 57(4), 781-797. https://doi.org/10.1111/0022-4537.00241 
Eagly, A. H., Wood, W. y Diekman, A. B. (2000). Social role theory of sex differences and similarities: A current appraisal. En T. Eckes y H. M. Trautner (Eds.), The developmental social psychology of gender (pp. 123-174). Mahwah: Erlbaum.

ETAN. (2000). Política científica de la Unión Europea: Promover la excelencia mediante la integración de la igualdad entre géneros. Bruselas: Comisión Europea.

Ferreyra, A. y De Longhi, A. L. (2014). Metodología de la investigación I. Córdoba: Encuentro Grupo Editorial.

García De León, M. A. y García, M. (2001). Las académicas. Profesorado universitario y género. Madrid: Instituto de la Mujer, Ministerio de Trabajo y Asuntos Sociales.

García, M. M., Jiménez, M. L. y Martínez, E. (2010). Guía para incorporar la perspectiva de género a la investigación en salud. Recuperado de http://www.umaza.edu.ar/archivos/file/GuiaInvestigacionGenero.pdf

Goetz, J. P. y Lecompte, M. D. (1988). Etnografía y diseño cualitativo en investigación educativa. Madrid: Morata.

Hernández, R., Fernández, C. y Baptista, P. (2003). Metodología de la investigación. Ciudad de México: McGraw- Hill.

Laorden, A. y Giménez, P. (1978). La mujer en la universidad española. Papers: Revista de Sociologia, 9, 73-88. https://doi.org/10.5565/rev/papers/v9no.1000

Lavié, J. M. (2009). El liderazgo a debate: nuevas perspectivas sobre un viejo conocido. En M. Sánchez-Moreno (Ed.), Mujeres dirigentes en la universidad: Las texturas del liderazgo. (pp. 59-79). Zaragoza: Sagardiana.

Lee, R. M. (2000). Unobtrusive methods in social research. Buckingham: Open University Press.

Ley Orgánica 4/2007, de 12 de abril, por la que se modifica la Ley Orgánica 6/2001, de 21 de diciembre, de Universidades. Boletín oficial del Estado, núm. 89, Viernes 13 abril 2007, 16241-16260. Recuperado de https://www.boe.es/boe/dias/2007/04/13/pdfs/A1624116260.pdf

Ley orgánica 14/2011, de 1 de junio, de la Ciencia, la Tecnología y la Innovación. Boletín oficial del Estado, núm.131, Jueves 2 de junio de 2011, 54387- 54455. Recuperado de https://www.boe.es/boe/dias/2011/06/o2/pdfs/BOE-A-2011-9617.pdf

Lyness, K. y Heilman, M. (2006). When fit is fundamental: Performance evaluations and promotions of upper-level female and male managers. Journal of Applied Psychology, 91(4), 777-785. https://doi.org/10.1037/0021-9010.91.4.777

Massachusetts Institute of Technology. (1999). A study on the status of women faculty in science at MIT. Boston, MA: MIT.

Matus-López, M. y Gallego-Morón, N. (2015). Techo de cristal en la universidad. Si no lo veo no lo creo. Revista Complutense de Educación, 26(3), 611-626. https://doi.org/10.5209/rev_RCED.2015.v26.n3.44491

MECD. Ministerio de Educación, Cultura y Deporte. (2016). Datos y cifras del sistema universitario español Curso 2015-2016. Recuperado de http://www.mecd.gob.es/dms/mecd/serviciosal-ciudadano mecd/estadisticas/educacion/universitaria/datos-cifras/datos-y-cifras-SUE2015-16-web-.pdf

MEC. Ministerio de Educación y Ciencia. (2007). Académicas en cifras. Recuperado de http://www.uned.es/rectoras/2007-academicas-en-cifras.pdf 
Morrison, A. M., White, R.P. y Van Velsor, E. (1987). Breaking the glass ceiling: Can women reach the top of america's largest corporations? Nueva York, NY: Addison-Wesley.

Pastor, I. (2008). Dones i homes a la URV: Un estudi de les desigualtats per raó de gènere. Tarragona: Publicacions Universitat Rovira i Virgili.

Pérez, F. y Peiró, J. M. (1999). El sistema de gobierno de la universidad española. Recuperado de http://www.uv.es/perezgar/publicaciones/gob-univ.pdf

Sabariego-Puig, M., Massot-Lafon, I. y Dorio-Alcaraz, I. (2009). Métodos de investigación cualitativa. En R. Bisquerra (Ed.), Metodología de la investigación educativa (pp. 293-328). Madrid: La Muralla.

Sánchez-Moreno, M., López-Yáñez, J. y Altopiedi, M. (2014). Liderazgo en la universidad: Perfil de mujeres y hombres gestores académicos. REICE. Revista Iberoamericana sobre Calidad, Eficacia y Cambio en Educación, 12(5), 21-42.

Sánchez de Madariaga, I., de la Rica, S. y Dolado, J. J. (2011). Libro blanco. Situación de las mujeres en la ciencia española. Madrid: Unidad de Mujeres y Ciencia, Ministerio de Ciencia e Innovación.

Webb, E. J., Campbell, D. T., Schwartz, R. D. y Sechrest, L. (2000). Unobtrusive measures. Thousand Oaks, CA: Sage Publications Inc.

Wenneras, C. y Wold, A. (1997). Nepotism and sexism in peer-review. Nature, 387, 341-343. https://doi.org/10.1038/387341a0

\section{Breve CV de las autoras}

\section{Itziar Rekalde Rodríguez}

Profesora agregada en el Departamento de Didáctica y Organización Escolar de la Universidad del País Vasco, y Coordinadora del Servicio de Asesoramiento Educativo en el Campus de Gipuzkoa, Vicerrectorado de Estudios de Grado e Innovación. Doctora en Pedagogía por la Universidad de Salamanca. Actualmente desarrolla su labor docente e investigadora en la formación inicial y continua del profesorado universitario, concretamente en el diseño, desarrollo y evaluación de procesos formativos. ORCID ID: 0000-0002-9297-7634. Email: itziar.rekalde@ehu.eus

\section{Esther Cruz Iglesias}

Profesora en el Departamento de Didáctica y Organización Escolar. Doctora por la Universidad del País Vasco con la tesis titulada "Las competencias del profesorado universitario: Un enfoque desde la perspectiva del alumnado. Análisis de un centro". Tesis defendida en el 2016. ORCID ID: 0000-0001-6093-9309. Email: esther.cruz@ehu.eus 